\title{
Introduction to the Artificial Intelligence and Big Data Analytics Management, Governance, and Compliance Minitrack
}

\author{
Michael Goul \\ W. P. Carey School of Business \\ Arizona State University \\ Michael.Goul@asu.edu
}

\author{
Jeffrey Saltz \\ School of Information Studies \\ Syracuse University \\ jsaltz@syr.edu
}

\author{
Anna Sidorova \\ College of Business \\ University of North Texas \\ anna.sidorova@unt.edu
}

\section{Introduction}

Artificial Intelligence (AI) and Big Data applications are becoming increasingly important strategic assets as they enable organizations to differentiate from their competitors by offering new data-driven products and services, by achieving increased agility in operations and decision making, by enhancing the discovery of new business insights, and by augmenting decision making and acting on decisions in a faster, more streamlined manner. As organizations become more reliant on AI and data-driven models for insight, decision making and action, they need new theories, frameworks and methodologies that can help them in the following areas:

- Deploying effective strategies and policies for managing AI and Big Data

- Streamlining processes to develop and deploy analytical models and Machine Learning (ML) algorithms

- Designing new KPIs and deploying actionable dashboards

- Managing and staffing AI, ML and data science teams

- Structuring analytics functions/capabilities within organizations

- Designing, staffing and providing direction to AI, data and analytics governance committees

- Managing AI and Big Data Analytics project and deployment risk and

- Advancing AI and Big Data capability maturity

What is new? Organizational roles like Chief Artificial Intelligence Officer, Chief Data Officer and Chief Analytics Officer are emerging. Funding models for prioritizing analytics opportunities are more frequently being discussed. Centers of Excellence and shared services entities are being created to handle and manage increasing data science and AI workloads. New agile methodologies are emerging, and building an organization-wide culture of evidence-based management is becoming a competitive necessity. AI and Big Data resource investment decisions are becoming more complex as there is increasing Internet of Things convergence within corporate IT portfolios.

Effective organizational management and governance of AI and big data analytics practices are necessary in order to mitigate risks and realize benefits. Organizations need to capture and manage critical metainformation detailing modeling and environmental assumptions underlying analytics solutions, and they need to establish policies and a culture designed to ensure adherence to the highest ethical standards of data management, and predictive model/AI deployments. In addition, organizations need new legal analytics tool familiarity, and they need to understand the role of legal analytics in compliance. Unleashing machine learning algorithms that may take on a life of their own can require safeguards and risk mitigation monitoring.

\section{Emerging Research Issues}

AI Governance, in particular, has become an important topic in many industries and across many application areas. Companies like Google have established principles for responsible AI technology development. The company also suggests needed government and standards organizations' action in areas including explainability, fairness appraisal, safety considerations, human-AI collaboration and liability frameworks. A wide variety of disciplines are also proposing specific research agendas around AI governance, yet there is little discussion for how these different research streams might be integrated. Similarly, there are different regulatory approaches underway in different global regions and countries.

With the coining of the phrase, the "AI Powered Organization," many suggest that rapid and revolutionary change will be required. This means that AI management processes and governance approaches 
will be inextricably linked as deployments scale. Research agendas that align management issues and governance approaches will be key. Similarly, nonlinear scaling will imply that AI, Big Data analytics and traditional IT system interactions could lead to unintended consequences for deploying organizations. Connecting the dots on governance and management of a broadening portfolio of technological capabilities will require new ways of organizing, monitoring and recovering.

The host of technological, process, people, governmental and societal issues surrounding increasing trust in and dependence on Big Data Analytics and AI is staggering. In general, IT governance in organizations has often failed, many compliance programs have failed, and IT management, particularly IT project management, has experienced a fair share of failures. One of the most important goals for new research is to help mitigate the risk of failures across the AI and Big Data analytics management, governance and compliance sea-changes already underway.

\section{Sessions}

This mini track examines challenges surrounding theory, frameworks, development, evaluation, and impact of AI, Big Data, data science and analytics management, governance, and compliance. The papers accepted for the mini track investigate these issues in different ways.

The first paper, titled "Artificial Intelligence Regulation: A Meta-Framework for Formulation and
Governance" by Patricia Almeida, Josivania Silva Farias and Carlos Santos, presents a meta-framework for Artificial Intelligence regulation that encompasses all stages of international public policy-making, from formulation to sustainable governance. The framework was developed via a systematic review of the literature on Artificial Intelligence Regulation. Their framework provides a trustworthy lens for societies to think collectively and make informed policy decisions related to what, when, and how the uses and applications of Artificial Intelligence should be regulated.

In the second paper titled "Preventing Algorithmic Bias in the Development of Algorithmic Decision-Making Systems: A Delphi Study", by Banu Aysolmaz, Deniz Iren, and Nancy Dau, the authors explore automated decisions made about or on behalf of us by Algorithmic Decision-Making (ADM) systems. While ADM systems can provide promising business opportunities, their implementation can pose numerous challenges. For example, algorithmic bias could result in systematical discrimination and unfair decisions by favoring certain individuals over others. While several approaches have been proposed to correct erroneous decision-making in the form of algorithmic bias, these proposed remedies have mostly dealt with identifying algorithmic bias after the unfair decision has been made rather than preventing it. In this study, the authors used the Delphi method to propose an ADM systems development process that identifies sources of algorithmic bias at each step of the process. The results of this research can help pave the way to achieve fair and trustworthy ethics-by-design ADM systems. 